\title{
Seminal fluid mediates ejaculate competition in social insects
}

Den Boer, Susanne Petronella A; Baer, Boris; Boomsma, Jacobus Jan

Published in:

Science

DOI:

10.1126/science.1184709

Publication date:

2010

Document version

Early version, also known as pre-print

Citation for published version (APA):

Den Boer, S. P. A., Baer, B., \& Boomsma, J. J. (2010). Seminal fluid mediates ejaculate competition in social insects. Science, 327(5972), 1506-1509. https://doi.org/10.1126/science.1184709 
The implication of our results for dynamic combinatorial chemistry is that this technique need not be limited to exploring the thermodynamic minima of molecular networks; kinetic control can dominate, provided that the noncovalent interactions are sufficiently strong and/or numerous. Our results further show that it is possible to obtain kinetic products from an assembly process where all the individual steps (covalent disulfide exchange and noncovalent peptide-peptide interactions) are reversible. Such a transition from thermodynamically controlled self-assembly to kinetic control must have been an important step in the origin of life, as life is far from equilibrium (35). Finally, our approach represents a promising method for the discovery of self-synthesizing materials in general and noncovalent polymers in particular (36).

\section{References and Notes}

1. L. E. Orgel, Nature 358, 203 (1992).

2. L. E. Orgel, Acc. Chem. Res. 28, 109 (1995).

3. E. Szathmáry, ]. Maynard Smith, J. Theor. Biol. 187, 555 (1997).

4. G. von Kiedrowski, Angew. Chem. Int. Ed. Engl. 25, 932 (1986).

5. G. von Kiedrowski, B. Wlotzka, ]. Helbing, M. Matzen, S. Jordan, Angew. Chem. Int. Ed. Engl. 30, 423 (1991)

6. N. Paul, G. F. Joyce, Proc. Natl. Acad. Sci. U.S.A. 99, 12733 (2002).
7. D. H. Lee, J. R. Granja, J. A. Martinez, K. Severin, M. R. Ghadri, Nature 382, 525 (1996).

8. S. Yao, I. Ghosh, R. Zutshi, ]. Chmielewski, J. Am. Chem. Soc. 119, 10559 (1997).

9. A. Vidonne, D. Philp, Eur. J. Org. Chem. 2009, 593 (2009).

10. T. Tjivikua, P. Ballester, J. Rebek, J. Am. Chem. Soc. 112 1249 (1990).

11. V. Rotello, ]. I. Hong, ]. Rebek, J. Am. Chem. Soc. 113 9422 (1991).

12. B. Wang, I. O. Sutherland, Chem. Commun. (Camb.) 16, 1495 (1997).

13. R. J. Pieters, I. Huc, ]. Rebek, Angew. Chem. Int. Ed. Engl. 33, 1579 (1994).

14. D. H. Lee, K. Severin, Y. Yokobayashi, M. R. Ghadiri, Nature 390, 591 (1997).

15. P. T. Corbett et al., Chem. Rev. 106, 3652 (2006)

16. S. Xu, N. Giuseppone, J. Am. Chem. Soc. 130, 1826 (2008).

17. R. Nguyen, L. Allouche, E. Buhler, N. Giuseppone, Angew. Chem. Int. Ed. 48, 1093 (2009).

18. J. W. Sadownik, D. Philp, Angew. Chem. Int. Ed. 47, 9965 (2008).

19. R. J. Williams et al., Nat. Nanotechnol. 4, 19 (2009).

20. W. F. DeGrado, J. D. Lear, J. Am. Chem. Soc. 107, 7684 (1985)

21. Y. Krishnan-Ghosh, S. Balasubramanian, Angew. Chem. Int. Ed. 42, 2171 (2003)

22. S. Otto, R. L. E. Furlan, ]. K. M. Sanders, J. Am. Chem. Soc. 122, 12063 (2000)

23. Materials and methods are available as supporting material on Science Online.

24. N. J. Greenfield, Nat. Protoc. 1, 2876 (2007).

25. H. LeVine III, Protein Sci. 2, 404 (1993).

26. H. Puchtler, F. Sweat, M. Levine, J. Histochem. Cytochem. 10, 355 (1962)
27. W. E. Klunk, J. W. Pettegrew, D. J. Abraham, ]. Histochem. Cytochem. 37, 1273 (1989).

28. J. Kong, S. Yu, Acta Biochim. Biophys. Sin. (Shanghai) 39, 549 (2007).

29. Reports of molecular systems whose mechanosensitivity affects defined covalent transformations are rare. For examples of such systems, see (30-32).

30. C. R. Hickenboth et al., Nature 446, 423 (2007).

31. D. A. Davis et al., Nature 459, 68 (2009)

32. A. Piermattei, S. Karthikeyan, R. P. Sijbesma, Nat. Chem. 1, 133 (2009).

33. A. T. Petkova et al., Science 307, 262 (2005).

34. This estimate is based on the typical thickness of a layer of lubricant between sliding nonconformal contacts. See (37).

35. A. Pross, J. Theor. Biol. 220, 393 (2003).

36. L. Brunsveld, B. J. B. Folmer, E. W. Meijer, R. P. Sijbesma, Chem. Rev. 101, 4071 (2001).

37. R. S. Dwyer-joyce, B. W. Drinkwater, C. J. Donohoe, Proc. R. Soc. Lond. A 459, 957 (2003).

38. We thank ]. K. M. Sanders and ]. B. F. N. Engberts for critical reading of the manuscript and C. M. Dobson for useful discussions. This research has been supported by the Engineering and Physical Sciences Research Council, the Royal Society, the Dynamic Combinatorial Chemistry Marie-Curie Research Training Network, and Cooperation in Science and Technology (COST) CM0703.

Supporting Online Material

www.sciencemag.org/cgi/content/full/327/5972/1502/DC1 Materials and Methods

Figs. S1 to S12

References

1 October 2009; accepted 10 February 2010 $10.1126 /$ science. 1182767

\title{
Seminal Fluid Mediates Ejaculate Competition in Social Insects
}

\author{
Susanne P. A. den Boer, ${ }^{1}$ Boris Baer, ${ }^{2,3}$ Jacobus ]. Boomsma ${ }^{1 *}$
}

Queens of ants and bees normally obtain a lifetime supply of sperm on a single day of sexual activity, and sperm competition is expected to occur in lineages where queens receive sperm from multiple males. We compared singly mated (monandrous) and multiply mated (polyandrous) sister groups of ants and bees and show that seminal fluid of polyandrous species has a more positive effect on the survival of a male's own sperm than on other males' sperm. This difference was not observed in the monandrous species, suggesting that incapacitation of competing sperm may have independently evolved in both bees and ants. In Atta leafcutter ants, the negative effect of the seminal fluid of other males was negated by secretion from the queen sperm-storage organ, suggesting that queens may control ejaculate competition after sperm storage.

$\mathrm{M}$ uch sexual selection in polyandrous species occurs after mating in the form of sperm competition and cryptic female choice (1-3). In most animals, males seek additional mates to increase the number of their offspring, and females may remate to gain direct or indirect benefits to promote offspring

${ }^{1}$ Centre for Social Evolution, Department of Biology, University of Copenhagen, Universitetsparken 15, 2100 Copenhagen, Denmark. ${ }^{2}$ ARC Centre of Excellence in Plant Energy Biology, MCS Building M310, The University of Western Australia, 6009 Crawley, Australia. ${ }^{3}$ Centre for Evolutionary Biology, School of Animal Biology (M092), The University of Western Australia, 6009 Crawley, Australia.

*To whom correspondence should be addressed. E-mail: jjboomsma@bio.ku.dk quality [e.g., (1-5)]. The eusocial ants, bees, wasps, and termites, in which only relatively few individuals have the opportunity to mate, are exceptions, because they evolved from strictly monogamous ancestors $(6,7)$. Newly eclosed queens of ants, bees, and wasps are receptive for mating during a very brief period of time (a few hours to a few days) and never remate $(7,8)$. Whereas queens from basal lineages store only a single ejaculate, obligate multiple queen-mating has evolved secondarily in honeybees, vespine wasps, leafcutter ants, army ants, harvester ants, and a few minor taxa $(6,7,9)$. Thus, opportunities for postcopulatory sexual conflict have repeatedly emerged and may have induced convergent adaptive responses.
The absence of remating implies that ejaculates from multiple males coexist within a queen's sperm storage organ (spermatheca) throughout her life $(7,10)$. This situation is likely to have undergone selection for prudent mutual exploitation among partners, similar to mutualisms characterized by lifetime commitment $(11,12)$. Ejaculate competition might occur shortly after multiple insemination, if it has no major negative effects on queen health and longevity $(10,13)$ and leaves sufficient high-quality sperm for her to realize her lifetime reproductive potential. However, selection is expected to act against antagonistic interactions between ejaculates after sperm storage, because a female's reproductive life span ultimately depends on her ability to fertilize eggs from this nonrenewable stock of sperm (14).

Queens of Atta leafcutter ants use few sperm to fertilize each egg, consistent with a correlation between lifetime reproductive success and sperm-storage limitations (15). This implies that selection on sperm viability should be strong and that both the male seminal fluid and queen spermathecal fluid are necessary for reproductive success. Male accessory gland (AG) secretion confers a positive effect on sperm survival, even in very small quantities, in leafcutter ants (16) and honeybees (17), and the spermathecal fluid of honeybee queens also positively influences the viability of stored sperm (17).

We used an in vitro sperm survival assay (16-18) to test the effects of own AG secretion, AG secretion of other males, and queen 
spermathecal fluid on sperm survival in two species of corbiculate bees and three species of fungus-growing ants. This assay (18) provides relative sperm survival data that can be directly compared within experiments, but does not allow accurate comparisons of absolute sperm survival percentages across species, because sperm and seminal fluid contributions may vary across natural ejaculates (19).

Our choice of species exploited the sharp evolutionary transition shown by both the corbiculate bees and the fungus-growing ants from exclusive single mating of queens, i.e., storing sperm from a single ejaculate (Bombus terrestris bumblebees and Trachymyrmex cf. zeteki ants), to multiple mating, i.e., queens storing sperm from multiple males with which they mate in quick succession (Apis mellifera honeybees and Atta colombica and Acromyrmex echinatior leafcutter ants) $(6,20)$. We found that providing sperm with AG secretion from the same male significantly increased sperm survival in all five species (Fig. 1), indicating that sperm is consistently protected by the quantities of $\mathrm{AG}$ secretion that we used in our assays. The absolute sperm survival values are almost certainly underestimates for sperm viability in natural ejaculates, because the Hayes saline buffer used does not nourish sperm, so that higher AG concentrations may be needed to reach the close to $100 \%$ in vitro sperm survival that can be achieved with this assay (16).

We next investigated whether the $A G$ secretion of other conspecific males would be equally effective in enhancing sperm survival (18). We expected that AG secretions might be at least partially hostile to other males' sperm in the polyandrous species, but that no such effects should occur in their monandrous sister groups where ejaculates never interact in vivo. We used generalized estimating equations, nesting species within mating systems and using individuals as repeated measures (18), to compare the effects of AG secretions from a focal male, brothers of the focal male, and unrelated males on sperm survival. We found significant effects of mating system (polyandrous versus monandrous, $\chi^{2}=22.33$, df $\left.=1, P<0.001\right)$ and treatment (AG origin, $\chi^{2}=19.17, \mathrm{df}=2, P<$ $0.001)$. The overall mating system difference may not be biologically meaningful because the absolute sperm survival percentages across species (nested within mating system) are not directly comparable. However, a significant interaction between mating system and treatment $\left(\chi^{2}=18.62, \mathrm{df}=2, P<0.001\right)$ indicated that sperm exposed to alien seminal fluid showed reduced levels of sperm survival in polyandrous species, but not in monandrous species (Fig. 2). This was confirmed by a rerun of the analysis for the monandrous species separately, where no significant effect of treatment could be detected $\left(\chi^{2}=2.13, \mathrm{df}=2, P=\right.$ 0.346 for Bombus and $\chi^{2}=0.02$, df $=1, P=$ 0.883 for Trachymyrmex). The origin of nonself
Fig. 1. Positive effects of male AG secretion on sperm survival across three species of fungusgrowing ants (Trachymyrmex cf. zeteki, Acromyrmex echinatior, Atta colombica), a bumblebee (Bombus terrestris), and a honeybee (Apis mellifera). The upper five charts show the absolute sperm viability values in the control treatments with Hayes saline (left) and the AG supplementations (right) that produced the AG/control survival ratios in the lower panel. The data for $A$. colombica and $A$. mellifera are from previous studies $(15,16)$, whereas those for the other three species were collected in the present study. The slope of the regression in the lower panel $\left(y=0.824 x+0.784 ; r^{2}=0.876\right.$; $P=0.019$ ) is not significantly different from 1 (i.e., approximately parallel to the diagonal: 95\% confidence interval slope: 0.254-1.395). The intercept of the regression is significantly dif-
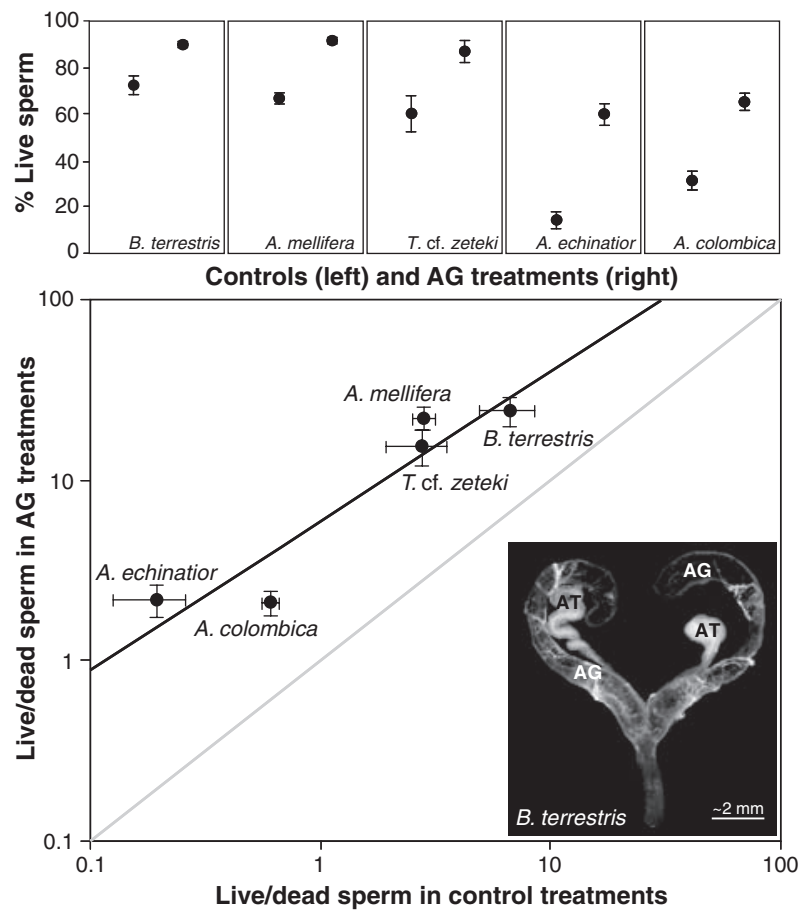

ferent from 1 (zero after log-transformation: $t_{3}=7.196 ; P=0.006$ ), indicating that the AG treatment had a consistently positive effect relative to control exposure to Hayes saline. The inset shows the male AGs and accessory testes (ATs) of a representative species (B. terrestris) as they were dissected. All bars are SEM.
Fig. 2. The effect of own, related (brother), and unrelated male AG secretion on sperm survival (mean $\pm \mathrm{SEM}$ ) in the monandrous bumble bee $B$. terrestris and fungus-growing ant Trachymyrmex cf. zeteki, and the polyandrous honeybee $A$. mellifera and fungus-growing ants $A$. echinatior and A. colombica. Photos of males are given in the panels. The brother treatment could not be performed for Trachymyrmex cf. zeteki (18). Those bars labeled with different letters ( $a$ or $b$ ) differ significantly (all $P<$ 0.001), whereas those with the same label do not (all $P>0.1$ ).

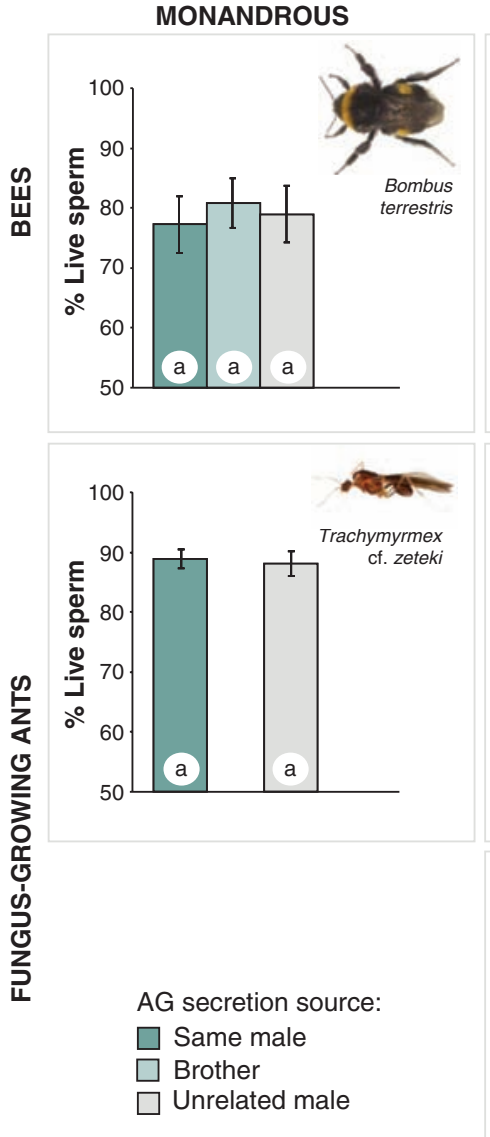

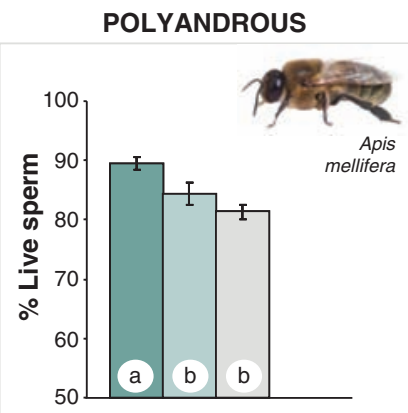

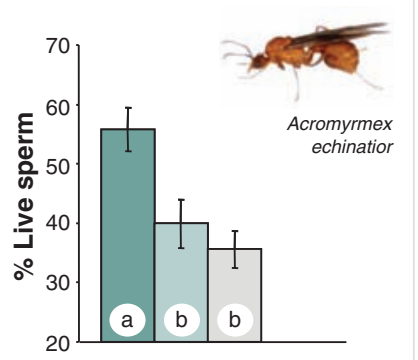

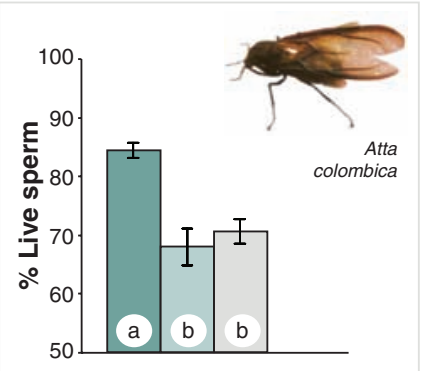


AG secretion, a brother or an unrelated male, did not differentially affect sperm survival $\left(\chi^{2}=\right.$ 2.44, $\mathrm{df}=1, P=0.118$ for Apis; $\chi^{2}=1.16, \mathrm{df}=$ $1, P=0.282$ for Acromyrmex; and $\chi^{2}=0.25$, df $=1, P=0.616$ for Atta). This suggests that the negative effects on sperm survival are triggered by a self versus nonself recognition process

These results are consistent with some form of ejaculate competition in polyandrous honeybees and leafcutter ants, and suggest that AG secretions contain both compounds that are favorable for sperm survival (Fig. 1) and substances that may incapacitate sperm of competing males (Fig. 2). To assess the combined effect of these putative forces on sperm survival, we repeated the second experiment for the three polyandrous species (but without the brother treatment), while adding an equal mix of own and alien AG secretion as an additional treatment (18) (Fig. 3). Overall treatment effects were significant in all cases $\left(\chi^{2}=8.31, \mathrm{df}=2\right.$, $P=0.016$ for Apis; $\chi^{2}=7.26$, df $=2, P=$ 0.027 for Acromyrmex; and $\chi^{2}=10.82, \mathrm{df}=2$, $P=0.005$ for Atta). However, own AG secretion does not appear to offset the negative effects of alien AG secretion, as pure alien and mixed AG treatments always resulted in similar but reduced sperm survival compared to the pure own AG treatment (detailed statistics are in the legend to Fig. 3).

After $30 \mathrm{~min}$ of exposure to alien $\mathrm{AG}$ secretion, sperm survival was reduced by $6.6 \%$ in Apis, $14.7 \%$ in Atta, and $18.0 \%$ in Acromyrmex relative to treatment with own AG secretion (Fig. 2). However, the residence time of inseminated honeybee sperm in the bursa copulatrix and lateral oviducts before being stored or discarded is 40 to 90 hours (21), whereas ejaculates of $A$. colombica are probably transferred to the spermatheca almost immediately
(22). Because rival ejaculates of Atta have less time to compete before being stored, there may be more damage per minute of exposure. This is consistent with $A$. mellifera queens expelling some $95 \%$ of the sperm provided by 10 to 20 matings (21), whereas A. colombica queens store essentially all sperm provided by 2 to 5 matings $(22,23)$. Typical sperm prestorage time in A. echinatior is unknown, but is up to 5 hours in $A$. versicolor, where queens appear to store around $10 \%$ of inseminated sperm (24).

Because every sperm cell provides a potential fitness benefit after the sperm storage process is complete $(14,15)$, we expected queens to either prevent hostile AG secretion from being stored, or to neutralize antagonistic interactions between ejaculates shortly after they enter the spermatheca. We tested this by collecting virgin $A$. colombica queens and artificially inseminating them with Hayes solution to obtain spermathecal fluid suspensions (18). Exposure of freshly dissected sperm from male accessory testes to mixtures of spermathecal fluid and seminal fluid of another male showed that spermathecal fluid maintains sperm survival at normal levels (Fig. 4). Thus, we conclude that spermathecal fluid has the potential to prevent the negative effects of $\mathrm{AG}$ secretion on alien sperm.

These results reveal consistent postmating sexual selection and sexual conflict in eusocial insects. They complement work elucidating why multiple queen-mating evolved $(9,25-27)$ and show that ants and bees are suitable models for testing sperm competition theory. This is because males have no influence on the fate of ejaculates after mating, and sperm storage has two distinct phases: a provisional one, varying across species from seconds to a few days, during which sperm competition may serve
Fig. 3. Own AG secretion does not counteract the negative effects on sperm survival (mean \pm SEM) of the AG secretion of other males in polyandrous ants and bees: $A$. mellifera, A. echinatior, and $A$. colombica. Overall treatment effects were significant in all three cases (see text). Specific contrasts were not significant for pure alien AG secretion versus a mixture of own and alien AG secretion $\left(\chi^{2}=0.00\right.$, $\mathrm{df}=1, P=0.964$ for Apis; $\chi^{2}$ $=0.05, \mathrm{df}=1, P=0.823$ for Acromyrmex; and $\chi^{2}=0.40$, $\mathrm{df}=1, P=0.529$ for Atta). However, contrasts were significant for pure own versus pure alien AG secretion $\left(\chi^{2}=4.86\right.$, $\mathrm{df}=1, P=0.028$ for Apis; $\chi^{2}$

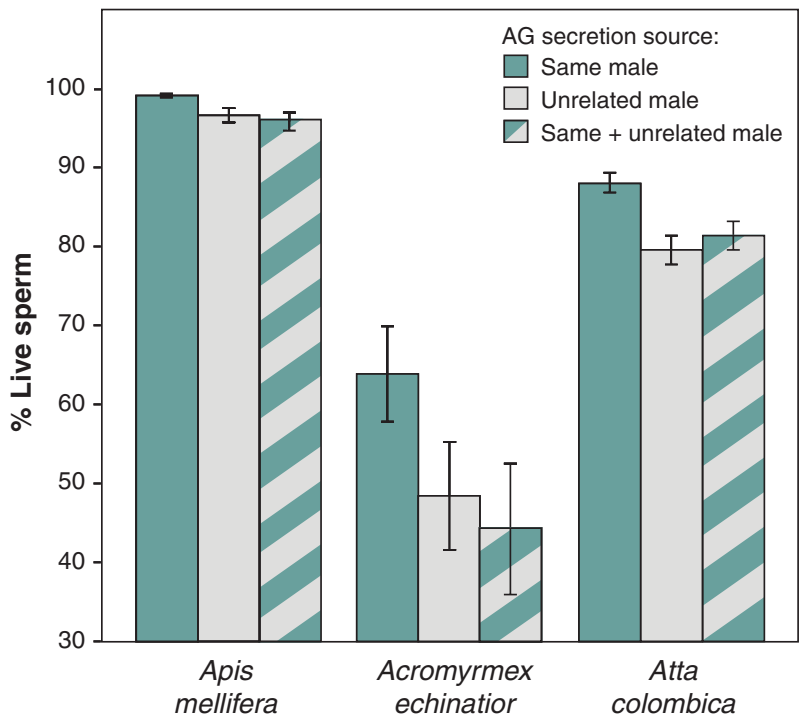

$=6.75, \mathrm{df}=1, P=0.009$ for Acromyrmex; and $\chi^{2}=9.25, \mathrm{df}=1, P=0.002$ for Atta), and for pure own versus a mixture of own and alien $A G$ secretion $\left(\chi^{2}=6.58, \mathrm{df}=1, P=0.010\right.$ for Apis; $\chi^{2}=$ 4.62, df $=1, P=0.032$ for Acromyrmex; and $\chi^{2}=9.89, \mathrm{df}=1, P=0.002$ for Atta). female interests; and a much longer permanent phase during which queens are expected to maximize the survival of all sperm. It therefore seems reasonable to expect that selection on male traits that enhance ejaculate competition is proportional to the duration of prestorage ejaculate interaction and to the ratio of inseminated versus long-term-stored sperm.

Antagonistic effects similar to those reported here may have arisen in other eusocial Hymenoptera that have evolved from single to multiple queen-mating $(6,25-27)$, and spermathecal fluids that neutralize aggressive male $A G$ compounds (compare to Fig. 4) may also be found in dwarf honeybees where sperm is directly transferred to the spermatheca (28), as in Atta (22). However, although phenotypic expressions of these sexual conflicts might be similar, their independent evolutionary origins suggest that the genes and pathways involved are different (7). Clarifying such molecular mechanisms is now feasible, as recent studies have shown that honeybee spermathecal proteomes differ appreciably from male AG proteomes $(17,29,30)$, consistent with functional differences connected with operating when sperm is in a state of long-term dormancy or metabolically active.

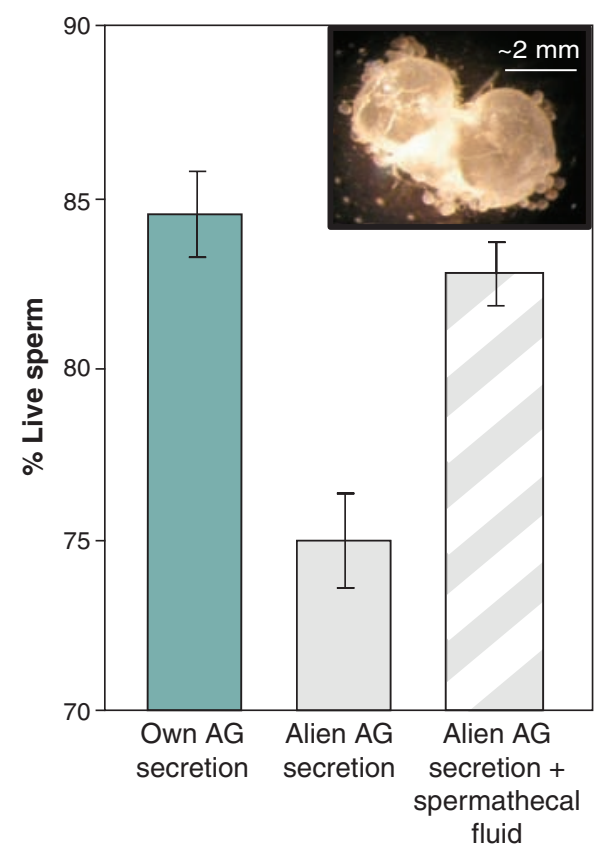

Fig. 4. Spermathecal fluid eliminates the negative effects of other males' AG secretions on sperm survival in $A$. colombica (mean \pm SEM). The inset shows a dissected spermatheca 1 hour after having been artificially inseminated with Hayes saline. The first two bars differ significantly $\left(\chi^{2}=15.04, \mathrm{df}=1, P<\right.$ $0.001)$, as do the second and third bar $\left(\chi^{2}=\right.$ 13.59, $\mathrm{df}=1, P<0.001$ ), but the bar on the right does not differ significantly from the bar toward the left $\left(\chi^{2}=2.38, \mathrm{df}=1, P=0.123\right)$. 


\section{References and Notes}

1. G. A. Parker, Biol. Rev. Camb. Philos. Soc. 45, 525 (1970)

2. W. G. Eberhard, Female Control: Sexual Selection by Cryptic Female Choice (Princeton Univ. Press, Princeton, N], 1996).

3. L. W. Simmons, Sperm Competition and Its Evolutionary Consequences in the Insects (Princeton Univ. Press, Princeton, N], 2001).

4. T. Chapman, G. Arnqvist, ]. Bangham, L. Rowe, Trends Ecol. Evol. 18, 41 (2003)

5. M. D. Jennions, M. Petrie, Biol. Rev. Camb. Philos. Soc. 75, 21 (2000)

6. W. O. H. Hughes, B. P. Oldroyd, M. Beekman, F. L. W. Ratnieks, Science 320, 1213 (2008).

7. J.J. Boomsma, Philos. Trans. R. Soc. Lond. Ser. B364, 3191 (2009)

8. B. Hölldobler, S. Bartz, in Experimental Behavioural Ecology and Sociobiology, B. Hölldobler, M. Lindauer, Eds. (Gustav Fischer Verlag, Stuttgart and New York, 1985), pp. 237-257.

9. J. J. Boomsma, D. J. C. Kronauer, J. S. Pedersen, in Organization of Insect Societies: From Genome to Sociocomplexity, ]. Gadau, ]. Fewell, Eds. (Harvard Univ. Press, Cambridge, MA, 2009), pp. 3-25.

10. J. J. Boomsma, B. Baer, ]. Heinze, Annu. Rev. Entomol. 50, 395 (2005).

11. D. K. Aanen et al., Science 326, 1103 (2009).

12. E. G. Leigh Jr., J. Evol. Biol. 23, 6 (2010).
13. T. Chapman, L. F. Liddle, J. M. Kalb, M. F. Wolfner, L. Partridge, Nature 373, 241 (1995).

14. B. Cole, Behav. Ecol. Sociobiol. 12, 191 (1983)

15. S. P. A. den Boer et al., Proc. R. Soc. B 276, 3945 (2009).

16. S. P. A. den Boer, ]. ]. Boomsma, B. Baer, Behav. Ecol. Sociobiol. 62, 1843 (2008)

17. S. P. A. den Boer, ]. ]. Boomsma, B. Baer, J. Insect Physiol. 55, 538 (2009).

18. Materials and methods are available as supporting material on Science Online.

19. L. Holman, Behav. Ecol. Sociobiol. 63, 1679 (2009).

20. P. Villesen, T. Murakami, T. R. Schultz, ]. ]. Boomsma, Proc. R. Soc. B. 269, 1541 (2002)

21. B. Baer, Apidologie 36, 187 (2005).

22. B. Baer, ]. ]. Boomsma, J. Morphol. 267, 1165 (2006).

23. B. Baer, S. A. O. Armitage, ]. J. Boomsma, Nature 441 872 (2006)

24. A. Reichardt, D. Wheeler, Behav. Ecol. Sociobiol. 38, 219 (1996).

25. J. J. Boomsma, F. L. W. Ratnieks, Philos. Trans. R. Soc. $B$ 351, 947 (1996)

26. ]. E. Strassmann, Insectes Soc. 48, 1 (2001).

27. R. H. Crozier, E. J. Fjerdingstad, Ann. Zool. Fenn. 38, 267 (2001).

28. N. Koeniger, G. Koeniger, S. Wongsiri, Apidologie 20 , 413 (1989)
29. B. Baer, H. Eubel, N. L. Taylor, N. O'Toole, A. H. Millar, Genome Biol. 10, R67 (2009).

30. B. Baer, ]. L. Heazlewood, N. L. Taylor, H. Eubel, A. H. Millar, Proteomics 9, 2085 (2009).

31. We thank G. Nachman and D. R. Nash for statistical help L. Holman and D. R. Nash for discussion and comments, M. Stürup for collecting ants in the field, M. King for collecting part of the sperm viability data for honeybees, the honeybee keepers of Western Australia (Better Bees of WA) for providing the necessary bee material, the Smithsonian Tropical Research Institute in Panama for facilities and logistic support during fieldwork, and the Autoridad Nacional de Ambiente (ANAM) for issuing collecting and export permits for fungus-growing ants. Supported by a grant from the Danish National Research Foundation (to ].J.B.) and an Australian Research Council Discovery Project (DP 0878107) and Queen Elizabeth II Fellowship (DP 0770050) (to B.B.).

\section{Supporting Online Material}

www.sciencemag.org/cgi/content/full/327/5972/1506/DC1

Materials and Methods

References

16 November 2009; accepted 17 February 2010 10.1126/science. 1184709

\section{Patterns of Diversity in Marine Phytoplankton}

\author{
Andrew D. Barton, ${ }^{{ }^{*}}{ }^{*}$ Stephanie Dutkiewicz, ${ }^{1}$ Glenn Flierl, ${ }^{1}$ Jason Bragg, ${ }^{2} \dagger$ Michael J. Follows $^{1}$
}

Spatial diversity gradients are a pervasive feature of life on Earth. We examined a global ocean circulation, biogeochemistry, and ecosystem model that indicated a decrease in phytoplankton diversity with increasing latitude, consistent with observations of many marine and terrestrial taxa. In the modeled subpolar oceans, seasonal variability of the environment led to competitive exclusion of phytoplankton with slower growth rates and lower diversity. The relatively weak seasonality of the stable subtropical and tropical oceans in the global model enabled long exclusion time scales and prolonged coexistence of multiple phytoplankton with comparable fitness. Superimposed on the decline in diversity seen from equator to pole were "hot spots" of enhanced diversity in some regions of energetic ocean circulation, which reflected lateral dispersal.

I n both marine and terrestrial environments, many taxa exhibit a decline in species diversity with increasing latitude $(1,2)$, and this pattern has important implications for ecosystem structure and function (3). The extent to which and why marine phytoplankton may follow such patterns is not yet clear, although it has been argued that the biogeography of microbes is governed by a similar set of processes as for macroorganisms (4). There is some evidence of latitudinal diversity gradients among certain taxa of marine microbes, including bacterioplankton $(5,6)$ and coccolithophorids $(7,8)$, although the generality

${ }^{1}$ Department of Earth, Atmospheric, and Planetary Sciences, Massachusetts Institute of Technology, 77 Massachusetts Avenue, Cambridge, MA 02139, USA. ${ }^{2}$ Department of Civil and Environmental Engineering, Massachusetts Institute of Technology, 77 Massachusetts Avenue, Cambridge, MA 02139, USA.

*To whom correspondence should be addressed. E-mail: adbarton@mit.edu

†Present address: Commonwealth Scientific and Industrial Research Organisation (CSIRO) Plant Industry, Canberra, ACT 2601, Australia. of these patterns, particularly in the open ocean,

In a recent study, a three-dimensional and timevarying global ocean circulation, biogeochemistry, and ecosystem model was initialized with a relatively large number (78) of virtual phytoplankton types whose traits were assigned stochastically from plausible ranges of possibilities (10-12). The modeled phytoplankton communities "self assembled" according to the relative fitness of the phytoplankton types in the regionally and seasonally varying resource and predatory environment. The emergent phytoplankton populations captured the observed large-scale oceanic patterns in the distribution of phytoplankton biomass and community structure, including the observed niche differentiation among ecotypes of the cyanobacterium Prochlorococcus in the Atlantic Ocean (11).

Here, we have studied an ensemble of 10 integrations of the global model, each member having a different, stochastically seeded selection of phytoplankton types, to examine and interpret is, as yet, equivocal $(9,10)$. the emergent patterns of phytoplankton diversity. In each of the solutions, after a decade of integration, a dozen or so phytoplankton types account for more than $99 \%$ of the total global phytoplankton biomass. Others persist at low abundance or with limited geographic distribution, and some decline toward virtual extinction. Fast-growing "opportunist" phytoplankton tend to dominate the biomass of the variable high latitudes, whereas "gleaners" (those best able to survive on minimal resources) dominate the stable, low-latitude seas $(12,13)$. There is also a degree of local coexistence among phytoplankton types. On an annual, vertically averaged basis, the phytoplankton diversity in the euphotic zone (here assumed to be 0 - to $260-\mathrm{m}$ depth) is lower in the polar and subpolar oceans and higher in tropical and subtropical latitudes (Fig. 1A). This meridional gradient is clearly seen in the zonally averaged view (Fig. 1B) and is consistent with numerous observations of marine and terrestrial ecosystems $(1,2)$, including the sparse observations of marine microbial diversity (5-8). Superimposed on the model's meridional gradient are "hot spots" of highest diversity, which are generally associated with regions of energetic circulation such as the western boundary currents. The Atlantic Ocean hot spots appear to be consistent with observations of increased diatom diversity near the North African and South American coasts (8).

The mechanisms for maintaining the diversity of life on Earth have long interested ecologists $(14,15)$, and the explanations for the meridional diversity gradient have been classified as historical, evolutionary, or ecological in nature $(6,16)$. Historical explanations invoke events and changes in Earth history, such as Milankovitch cycles, in setting current species diversity. Evolutionary explanations examine the rates of speciation and extinction and their balance through time $(17,18)$. These processes are not resolved in 